\title{
ESTRATÉGIA DE MITIGAÇÃO CONTRA COLISÃO DE OBJETOS ESPACIAIS COM A TERRA: DISTRIBUIÇÃO DE ÓRBITAS COM SISTEMA DE PROPULSÃO PLASMA
}

\author{
Thamis Côrtes Freire de Carvalho Ferreira ${ }^{1}$; Antônio Delson C. de Jesus ${ }^{2}$; \\ 1. Bolsista PIBIC-FAPESB, Graduando em Física, Universidade Estadual de Feira de Santana, \\ email: tham_mia@hotmail.com \\ 2. Orientador, Departamento de Física, Universidade Estadual de Feira de Santana, email: \\ a1d1j1@gmail.com
}

PALAVRAS-CHAVE: Estratégia, Mitigação, Propulsão Plasma

\section{INTRODUÇÃO}

Em meados do século XX o estudo da Dinâmica Orbital tornou-se fundamental para se atingir objetivos importantes nas missões espaciais em todo mundo. Estas missões, envolvendo diversos tipos de manobras e veículos espaciais, atendem a muitos objetivos científicos. É conhecido que o ambiente espacial está cercado de corpos que descrevem as mais diferentes e peculiares orbitas. Alguns deles apresentam a característica de terem suas evoluções orbitais muito próximas a órbita da Terra. A esses corpos damos o nome de (objetos espaciais próximos da Terra) ou NEO.

Modelos de observação tem buscado determinar as dimensões e as variações de proximidade desses objetos em relação a Terra. Resultados expostos por (Rabinowitz Et a1, 2000) mostram que para uma região de estudo foram observados 20 NEO que apresentam diâmetros maiores do que $5 \mathrm{Km}$, cerca de 1500 foram catalogados como maiores do que $1 \mathrm{Km}$ e um número superior a 130.000 apresentaram dimensões superiores a 100m. Conhecendo os riscos de iminente colisão desses corpos com a Terra, medidas de emergência precisam ser criadas para uma possível interação com esses objetos, a essas intervenções dá-se o nome de mitigação. Dentre essas estratégias o impacto cinético que visa alterar a órbita do NEO através do envio de uma ou mais espaçonaves com carga útil muito grande para impactar diretamente o objeto em alta velocidade.

A eficácia desta estratégia depende não apenas da massa do alvo, mas também em qualquer melhoria liquida, resultante do material que está sendo jogado para fora do alvo, na direção oposta a da carga útil, após o impacto. Nesse ambiente de manobras de mitigação a busca por um sistema de propulsão cada vez mais eficiente tem sido o desafio de muitos cientistas e pesquisadores. A propulsão elétrica (plasma) tem se mostrado eficiente, mais econômica e viável nas missões espaciais das últimas décadas. 


\section{MATERIAL E MÉTODOS OU METODOLOGIA (ou equivalente)}

A metodologia aplicada a este estudo tratou-se da dedução das equações da dinâmica relativa entre os corpos envolvidos (principalmente, NEO, veículo espacial impactor e Terra), considerando as forças gravitacional e a de propulsão. Por se tratar de um problema envolvendo $\mathrm{N}$-corpos, fez-se necessário o uso de integradores numéricos. O integrador escolhido foi o RENBOUND, que é um pacote de software no qual integra o movimento das partículas sobre a ação tanto da gravidade como de força nãogravitacionais, além de ter diversas rotinas que envolve colisão entre os corpos. A propulsão, considerada plasma produzirá uma velocidade de exaustão dos gases não constante, que dependerá dos campos elétrico e magnético que produzirão o plasma no propulsor. Diante disto, as equações foram modificadas para cada possibilidade de modelo dos campos, que determinaram as faixas tecnológicas de atuação do propulsor do veículo impactor. Visando assim, manobras de mitigação bem-sucedidas em função dos parâmetros físicos estabelecidos.

Os materiais necessários foram computadores para simulação numérica de alto desempenho, livros didáticos e específicos da área de Dinâmica Orbital, Probabilidade e Estatística, softwares específicos para uso do tratamento dos dados obtidos.

\section{RESULTADOS E/OU DISCUSSÃO (ou Análise e discussão dos resultados)}

O estudo se concentrou na análise dos parâmetros referentes aos sistemas de propulsão do veículo, os sistemas de propulsão usados para esse trabalho foram o químico e o plasma por efeito Hall. Os gráficos da figura 1 mostram os resultados referentes aos estudos dos parâmetros em função do novo periélio da órbita do NEO em relação a Terra para o propulsor químico.
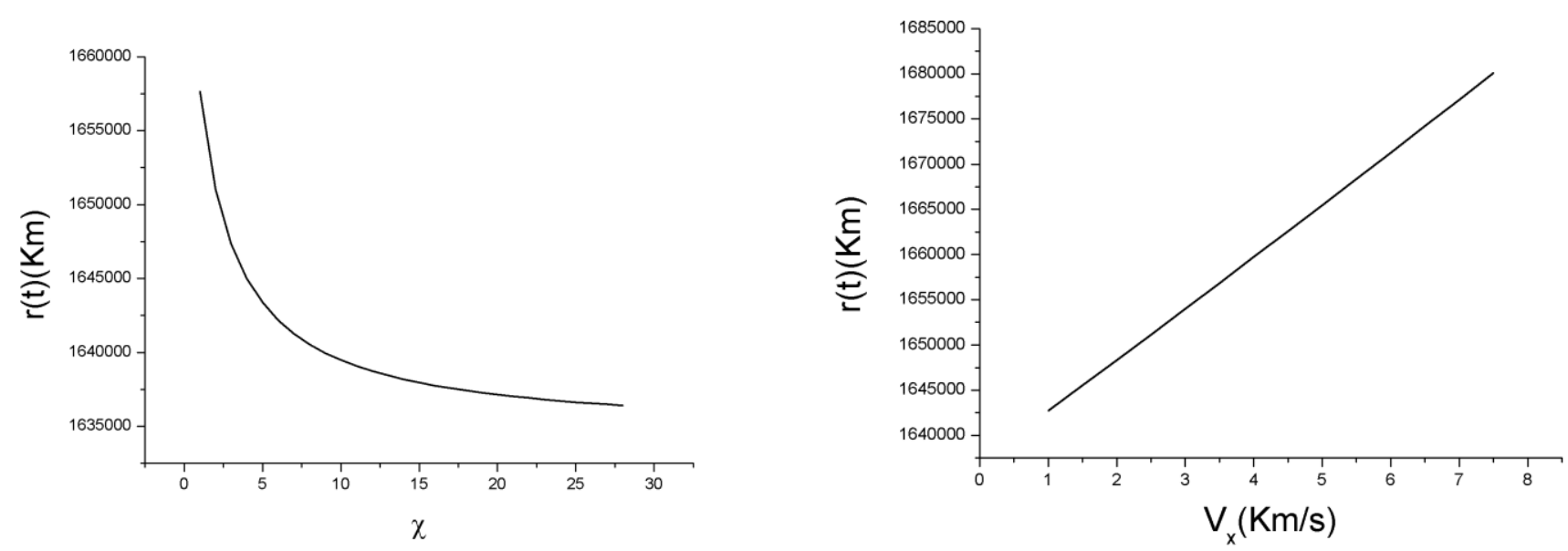

Figura 1-a) Distância relativa final versus fator de massa. b) Distância relativa final versus velocidade de exaustão na direção $x$. 
Os estudos obtidos permitiram determinar, através do estudo da magnetohidrodinâmica, as componentes das velocidades de exaustão para o propulsor plasma. Esses resultados encontram-se na equação ()

$$
\begin{gathered}
v_{x}=-\frac{\sigma}{\rho}\left(\frac{B_{t} B_{z}^{2} E_{o} \cos (\theta)}{|B|^{2}}\right) t \\
v_{y}=-\frac{\sigma}{\rho}\left(\frac{B_{t} B_{z}^{2} E_{o} \operatorname{sen}(\theta)}{|B|^{2}}\right) t \\
v_{z}=+\frac{\sigma}{\rho}\left(\frac{B_{t} B_{r} B_{z} E_{o}}{|B|^{2}}\right) t
\end{gathered}
$$

Com base nas expressões () juntamente com modelos apresentado por (JESUS, 2012) foi possível determinar as componentes da aceleração proveniente de um sistema de propulsão plasma com decaimento de massa exponencial:

$$
\begin{gathered}
a_{x}=-\left(\frac{v_{x y e^{-\gamma t}}}{\left(x+e^{-\gamma t}\right)}\right)-\dot{v}_{x} \ln \left(m_{o}\left(\chi+e^{-\gamma t}\right)\right) \\
a_{y}=-\left(\frac{v_{y} \gamma e^{-\gamma t}}{\left(\chi+e^{-\gamma t}\right)}\right)-\dot{v}_{y} \ln \left(m_{o}\left(X+e^{-\gamma t}\right)\right) \\
a_{z}=-\left(\frac{v_{z} \gamma e^{-\gamma t}}{\left(\chi+e^{-\gamma t}\right)}\right)-\dot{v}_{z} \ln \left(m_{\circ}\left(\chi+e^{-\gamma t}\right)\right)
\end{gathered}
$$

Após as simulações propostas foi possível determinar faixas para o campo elétrico e magnético do sistema de propulsão do veículo impactor que garantiam o evento colisional entre o veículo e o NEO. Os gráficos das figuras (2) e (3) apresentam as distâncias relativas no ponto do periélio da nova órbita obtida após a colisão em função dos parâmetros físicos referentes ao propulsor plasma.
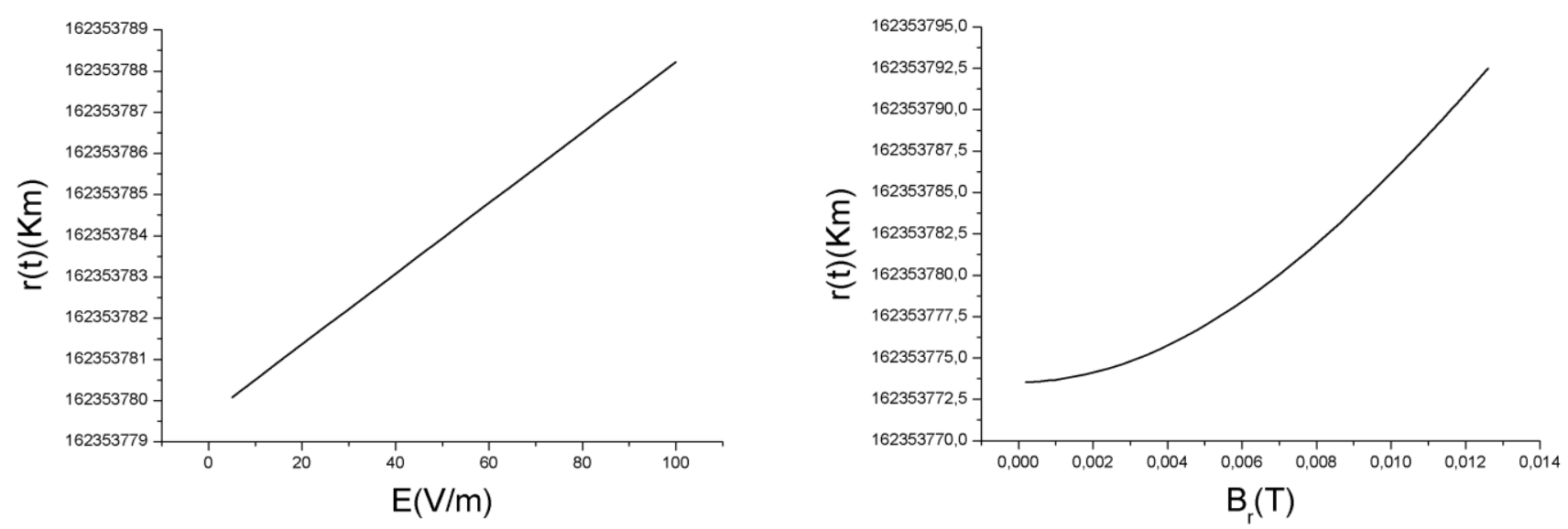

Figura 2 -a) Distância relativa final versus campo elétrico. b) Distância relativa final versus componente radial do campo magnético. 

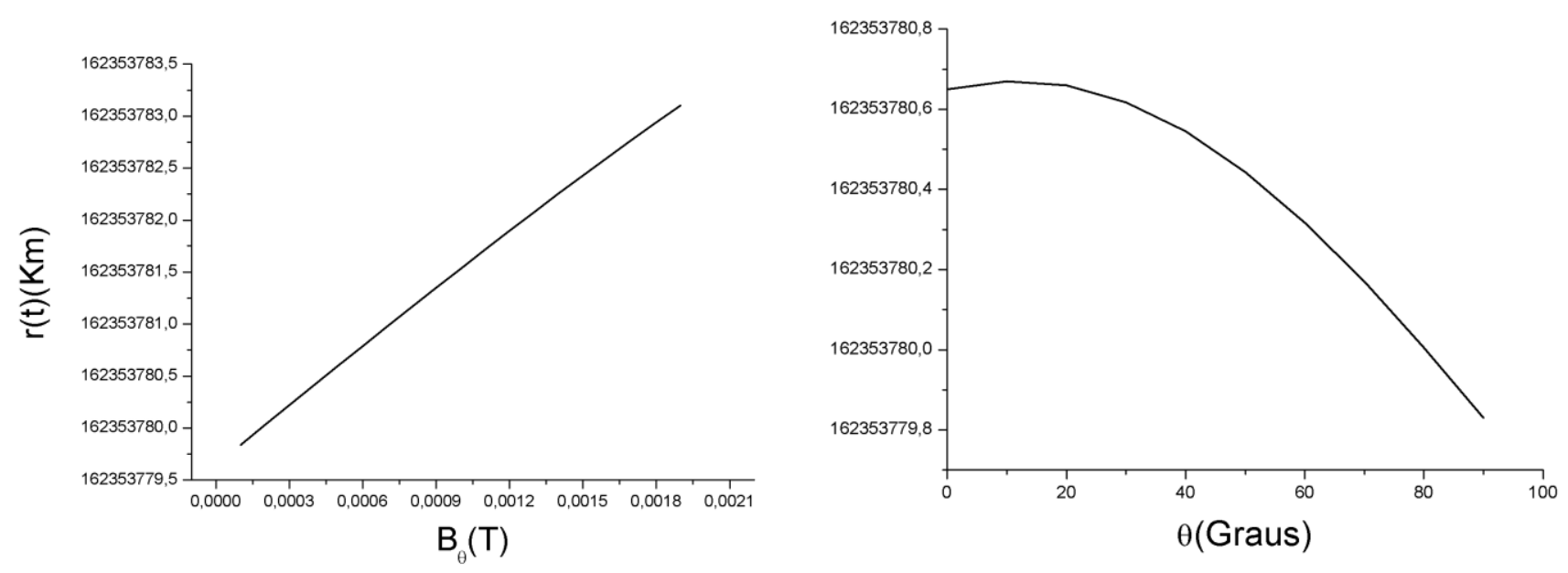

Figura 3 - a) Distância relativa final versus componente azimutal do campo magnético. b) Distância relativa final versus ângulo de projeção do campo magnético.

\section{CONSIDERAÇÕES FINAIS (ou Conclusão)}

O estudo do sistema de propulsão plasma demonstrou que para o campo elétrico, quando maior o valor aplicado sobre o campo maior é a distância relativa final observada, pois ele tem, entre suas funções, o papel de acelerar o fluxo de íons ao longo do canal de aceleração. Observou-se também o crescimento do perigeu da nova órbita em função do aumento no valor das componentes do campo magnético, sendo visto um crescimento linear para a componente azimutal e um crescimento exponencial para a componente radial e axial. Houve um decréscimo no valor da distância com o aumento do ângulo referente a projeção do campo magnético.

Deste modo a estratégia de mitigação por impacto cinético se mostrou bastante eficiente, pois foram obtidos valores consideravelmente grandes na órbita do NEO para o ponto mais próximo com Terra. Em relação aos sistemas de propulsão foi visto que a propulsão plasma se mostrou mais eficiente pois a distância relativa mínima (perigeu) em relação a Terra apresentou valores da ordem de $10^{8} \mathrm{Km}$ enquanto para a propulsão química os raios relativos finais foram da ordem de $10^{6} \mathrm{Km}$, para as condições apresentadas e para o objeto em estudo.

\section{REFERÊNCIAS}

JESUS, A.D.C.; Sousa, R. R.; Neto, E.V. Evasive Maneuvers in Route Collision with Space Debris Cloud. Journal of Physics: Conference Series 641 (2015)012021.

RABINOWITZ, David et al. A reduced estimate of the number of kilometre-sized nearEarth asteroids. Nature, v. 403, n. 6766, p. 165, 2000. 